\title{
Risk Factors for Group B Streptococcus Colonization and Drugs Sensitivity Pattern in a Nigerian Obstetric Population
}

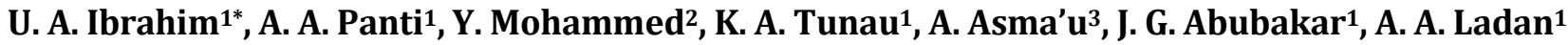 \\ ${ }^{1}$ Department of Obstetrics and Gynaecology, Usmanu Danfodiyo University Teaching Hospital, Sokoto, Nigeria \\ ${ }^{2}$ Department of Medical Microbiology, Usmanu Danfodiyo University Teaching Hospital, Sokoto, Nigeria \\ ${ }^{3}$ Department of Paediatrics, Usmanu Danfodiyo Univerrsity Teaching Hospitral, Sokoto, Nigeria \\ Email: *umariaugie@gmail.com, kapanti2002@gmail.com, yahyakt@yahoo.com, karimatunau@gmail.com, \\ karimatunau@gmail.com, jgarba80@gmail.com, ashiruladan@gmail.com
}

How to cite this paper: Ibrahim, U.A., Panti, A.A., Mohammed, Y., Tunau, K.A., Asma'u, A., Abubakar, J.G. and Ladan, A.A. (2021) Risk Factors for Group B Streptococcus Colonization and Drugs Sensitivity Pattern in a Nigerian Obstetric Population. Open Journal of Obstetrics and Gynecolo$g y, 11,804-814$

https://doi.org/10.4236/ojog.2021.116074

Received: April 23, 2021

Accepted: June 21, 2021

Published: June 24, 2021

Copyright $\odot 2021$ by author(s) and Scientific Research Publishing Inc. This work is licensed under the Creative Commons Attribution International License (CC BY 4.0).

http://creativecommons.org/licenses/by/4.0/

(c) (i) Open Access

\begin{abstract}
Background: Group B Streptococcus (GBS) is a major cause of bacterial infections in the perinatal period, of which colonization prevalence among Northern-Nigerian pregnant women is scarce. We attempted to determine 1) its prevalence, 2) risk factors for GBS colonization and 3) drugs-susceptibility. Methodology: This cross-sectional study involved 185 pregnant women between 35 - 37 weeks of gestation at tertiary health center of Sokoto, Nigeria. Vaginal/rectal swabs were collected, were cultured for GBS and tested for drugsusceptibilities. The study was conducted between December, 2017 and April, 2018. Results: One hundred and eighty five (185) pregnant women participated in this study. GBS vaginal-colonization-rate was 3.8\% (7/185). A significance relationship was observed between GBS-colonization and socio-economic class, as $57.10 \%(4 / 7)$ of the GBS positive women were of low-socio economic class ( $p$ 0.035). No associations were observed between GBS-colonization and the followings: maternal age, parity, poor obstetric outcome-history. All the 7 GBS positive cultures were sensitive to Clindamycin. One was sensitive to both Clindamycin and Ceftriaxone. None was sensitive to Penicillin. Conclusion: The prevalence of GBS colonization was low in this area. Maternal socio-economic class is found to be a risk of GBS-colonization.
\end{abstract}

\section{Keywords}

Group B Streptococcus, Risk Factors, Drugs Susceptibility

\section{Introduction}

Group B Streptococcus or Streptococcus agalactiae is a Gram positive Strepto- 
coccus [1]. It is a major cause of perinatal bacterial infections like amnionitis, bacteraemia, endometritis and urinary tract infections in pregnancy [2]. Group B Streptococcus can also ascend through the cervix without causing cervicitis and cross intact amniotic membranes causing in-utero fetal infection [3].

Approximately $10 \%-30 \%$ of women of child-bearing age carry GBS in the vaginal [2] [4] [5]. The prevalence may vary due to differences in the culture technique, the location, number of sites cultured and the population studied [6]. Maternal age and parity, marital status, education and smoking are factors that have been reported to influence the prevalence of GBS colonization [7]. The role of ethnicity and genetic factors are presumed since Caribbean Hispanics and black women were reported to be GBS carriers more frequently [8].

Prevalence and risk factors for GBS colonization among pregnant women varied from one region to another, Centre to Centre and even between ethnic groups. There was no similar study done in the study area. In our institution it has been observed that significant number of pregnant women presents with premature rupture of membrane (PROM), maternal fever in the advanced pregnancies and puerperal sepsis and there hasn't been any study to identify the causes. Information on the drugs sensitivity was alarming as some studies reported $100 \%$ sensitivity while some reported $100 \%$ resistance on same agents.

Approximately $10 \%-30 \%$ of women of child bearing age harbor GBS in their recto-vaginal compartment [2] [4] [5]. And GBS affects about 1 in every 2000 babies delivered in the United States [9]. A hospital based cross-sectional study in Pakistan, among 155 pregnant women between gestational age of 35 - 37 weeks on prevalence and risks factors revealed prevalence of $17 \%$ [10]. A study among 97 pregnant women at the Queen Elizabeth central hospital in Blantyre, Malawi showed prevalence of $16.5 \%$ [3], also prevalence of $28.8 \%$ have been reported in Uganda [11], 13.7\% in Ethopia [12], 2.5\% in Lome, Togo [13], 11.3\% in Ile-Ife [14] and $18.0 \%$ in Enugu [15]. However, studies from Ile-Ife and Enugu studied women between 35 - 40 and 24 - 37 weeks of gestation respectively.

There is paucity of data with regards to the risk factors for GBS colonization and drugs sensitivity pattern among pregnant women in Nigeria. Therefore, the aim of this study was to determine 1) prevalence of GBS, 2) risk factors for GBS colonization and 3) drug sensitivity pattern among pregnant women attending antenatal care clinic in UDUTH, Sokoto.

\section{Material and Method}

This study was a cross-sectional analytic study conducted among pregnant women at Usman Danfodiyo University Teaching Hospital (UDUTH), Sokoto. The desired sample size was calculated using prevalence formulae for estimating sample size in prevalence studies with a finite population correction as suggested by $\mathrm{Da}$ niels [16]. The prevalence used to calculate the sample size in this study was that of the study conducted by Onipede A et al. at Obafemi Awolowo University Teaching Hospital Complex, Ile-Ife, Nigeria on Group B Streptococcal Carriage 
During Late Pregnancy and Neonatal Infection [14]. A sample size 154 was calculated with attrition rate of $20 \%$ (30.8). A total of 185 women were used for this study, as follows;

Prevalence formulae for estimating sample size in prevalence studies was used with a finite population correction as suggested by Daniels [16]. The prevalence used to calculate the sample size in this study was that of the study conducted by Onipede et al. at Obafemi Awolowo University Teaching Hospital Complex, IleIfe, Nigeria on Group B Streptococcal carriage during late pregnancy and neonatal Infection. [4]

$$
n=\frac{Z_{1-q}^{2} / 2 P(1-P)}{d^{2}}
$$

where;

$p=$ Prevalence of GBS colonization in women delivering in Ile-Ife (11.3\%) [4]

$1-P=1$ minus the prevalence of GBS colonization in women

$Z_{1-a / 2}=$ standard errors associated with confidence interval of $95 \%$ (1.96)

$d=$ desired level of precision set to $5 \%$

$n=1.96^{2} \times[0.113(1-0.113)] / 0.05^{2}$

$n=154$

With attrition rate of $20 \%(30.8)=184.8$

A sample size of 185 women was used.

Women were selected via simple random sampling technique by balloting "YES" or "NO". Those who picked YES were recruited while those who picked NO were excluded from the study.

A semi-structured questionnaire was adopted from a similar study from IleIfe, with some modification. It was tested for validity and reliability at the state specialist hospital.

The patients' informed consents were sought for. Questionnaires were filled-in using information from the study participants including social-demographic and obstetrics characteristics.

The inclusion criteria was all pregnant women attending ANC at UDUTH who consented to participate in the study and are sure of their last menstrual period or had first trimester ultrasound scan. They were recruited at gestational age between 35 - 37 weeks and had their neonates delivered in our labour ward. Those excluded were pregnant women who had been on antibiotic treatment in the preceding two weeks prior to recruitment, those who had been diagnosed and/or are on management for premature rupture of membranes, antepartum haemorrhage and pre-eclampsia. Pregnant women who have diabetes or HIV in pregnancy were also excluded from the study.

Data was collected using a pre-coded semi-structured questionnaire administered to the study population by the principal investigator and research assistants and filled in promptly. Vaginal and rectal swabs were then taken per participant using two separate commercially available collection and transport system for both aerobes and anaerobes (Charcoal Swabs Amies Plastic Applicator 
with Rayon Tipped Black Cap, Stone, Staffs, UK) [8], the specimen was immediately transported to the Medical Microbiology laboratory of UDUTH, Sokoto for culture and susceptibility determination of the positive cultures. The swab samples were incubated aerobically at $37^{\circ} \mathrm{C}$ for 24 hours onto $5 \%$ Sheep Blood Agar (Oxoid England) and incubated overnight for evidence of haemolysis which suggest presence of GBS. Where there is evidence of haemolysis on the blood agar plate a quick gram stain smear was done. Where the Gram smear showed evidence of Gram positive cocci, it was then sub-cultured and CAMP (Christie, Artkins and Münch-Petersen) test was carried out from the primary plate using the pure colonies. Suspected GBS isolates were identified appropriately [16]. GBS antigen was confirmed definitively by serological grouping using Streptococcal group B reagent kit (Oxoid England) testing of selective broth [17].

The in-vitro sensitivity test of the isolates were determined in Mueller - Hinton agar plates by the standard method against penicillin $(10 \mu \mathrm{g})$, Cefotaxime (30 $\mu \mathrm{g})$ Erythromycin $(15 \mu \mathrm{g})$, Ceftriaxone $(30 \mu \mathrm{g})$, Vancomycin $(30 \mu \mathrm{g})$ and Clindamycin $(2 \mu \mathrm{g})$ and was interpreted according to the sensitivity pattern [1].

Data analysis was done using Statistical Package for Social Sciences (SPSS) version 20. Initial univariate analysis was used to calculate frequency distribution. Chi square was used to determine the risk factors associated with GBS colonization Level of significance was set at $p<0.05$.

\section{Ethical Consideration}

Ethical clearance was sought for from the Human Research and Ethical Committee of Usmanu Danfodiyo University Teaching Hospital, Sokoto with reference number: UDUTH/HREC/2017/No.621 before embarking on the study. The approval was signed by the committee chairman, Professor Nma M Jiya on the $19^{\text {th }}$ of September, 2017. International ethical principles according to the Helsinki declarations were considered during the course of the research. In addition, individual consent was also obtained from the study subjects before participation in the research.

\section{Result}

One hundred and eighty five participants were recruited for the study at both antenatal care clinic and pre-natal ward of UDUTH, Sokoto. Seven of the participants tested positive for GBS giving a prevalence of $3.8 \%$.

The mean age of the studied population was $28.49 \pm 5.6$ and $73 \%$ of the study participants were Hausa/Fulani. Majority (85.4\%) of the study participants were Muslims. And $78.4 \%$ were of monogamous family setting. Multi-parous women were $63.8 \%$ and $60 \%$ were of low socio-economic class.

Majority $(57.1 \%)$ of the GBS colonized women were between the ages of 30 39 years. None of those below the age of 20 years was GBS positive. However, there was no statistical significant relationship between maternal age and GBS colonization $\left(\chi^{2}=5.088, p=0.169\right)$. Most $(4 / 7)$ of the respondents $(57.1 \%)$ that 
were GBS positive were of low socio-economic status compared to the $18.5 \%$ of GBS negative group. Hence, there was statistical significant association between social-class and GBS colonization $\left(\chi^{2}=6.68, p\right.$ value $\left.=0.035\right)$. This is shown in Table 1 below.

With regards to the parity, majority of the GBS positive women were multi-gravidae and none of the primigravida was found to be GBS positive. However, there was no significant association between parity and GBS colonization $\left(\chi^{2}\right.$ $=2.227, p$ value 0.323). This is shown in Table 1 .

None of the women with GBS colonization had previous history of PROM or preterm delivery and there was no significant association between these factors and GBS colonization. This is shown in Table 2.

Similarly, none of the GBS colonized women had previous history of still-birth and there was no significant association between previous history of still-birth and GBS colonization ( $p$ value 0.653 ). This is shown in Table 2.

Table 1. Association between socio-demographic characteristics and GBS colonization.

\begin{tabular}{|c|c|c|c|c|}
\hline Characteristics & $\begin{array}{c}\text { GBS positive } \\
n(\%)\end{array}$ & $\begin{array}{c}\text { GBS negative } \\
\text { n (\%) }\end{array}$ & $\chi^{2} /$ Fischer exact & $p$ value \\
\hline \multicolumn{5}{|l|}{ Age (years) } \\
\hline Less than 20 & $0(0)$ & $9(5.1)$ & \multirow{4}{*}{5.088} & \multirow{4}{*}{0.169} \\
\hline 20 to 29 & $2(28.6)$ & $92(51.7)$ & & \\
\hline 30 to 39 & $4(57.1)$ & $74(41.6)$ & & \\
\hline 40 and above & $1(14.3)$ & $3(1.7)$ & & \\
\hline \multicolumn{5}{|l|}{ Occupation } \\
\hline Housewife & $3(42.9)$ & $94(52.8)$ & \multirow{4}{*}{1.535} & \multirow{4}{*}{0.706} \\
\hline Civil servant & $2(28.5)$ & $39(21.9)$ & & \\
\hline Trader & $1(14.3)$ & $32(18.0)$ & & \\
\hline Business & $1(14.3)$ & $13(7.3)$ & & \\
\hline \multicolumn{5}{|l|}{ Socio-economic class } \\
\hline Low & $4(57.1)$ & $33(18.5)$ & \multirow{3}{*}{6.68} & \multirow{3}{*}{0.035} \\
\hline Medium & $0(0.0)$ & $29(16.3)$ & & \\
\hline High & $3(42.9)$ & $116(65.2)$ & & \\
\hline \multicolumn{5}{|l|}{ Parity } \\
\hline Primigravida & $0(0.0)$ & $38(21.3)$ & \multirow{3}{*}{2.227} & \multirow{3}{*}{0.323} \\
\hline Multigravida & $5(71.4)$ & $113(63.5)$ & & \\
\hline Grand-multipara & $2(28.6)$ & $27(15.2)$ & & \\
\hline
\end{tabular}

Table 2. Association between previous bad pregnancy outcomes and GBS colonization.

\begin{tabular}{ccccc}
\hline Characteristic & $\begin{array}{c}\text { GBS Positive } \\
\text { N (\%) }\end{array}$ & $\begin{array}{c}\text { GBS Negative } \\
\text { N (\%) }\end{array}$ & $\begin{array}{c}\text { Statistics } \\
\text { Chi/Fischer }\end{array}$ & $p$ value \\
\hline $\begin{array}{c}\text { Previous PROM } \\
\text { Yes }\end{array}$ & $0(0.0)$ & $8(100.0)$ & 0.329 & 0.566 \\
No & $7(4.0)$ & $170(96.0)$ & & \\
Previous Preterm Delivery & & & & \\
Yes & $0(0.0)$ & $8(100.0)$ & 0.329 & 0.566 \\
No & $7(4.0)$ & $170(96.0)$ & & \\
Previous Stillbirth & & & & \\
Yes & $0(0.0)$ & $5(100.0)$ & 0.653 \\
No & $7(4.0)$ & $173(96.0)$ & & \\
\hline
\end{tabular}


All (100.00\%) of the GBS isolates were sensitive to Clindamycin and one of the isolates was sensitive to both the Clindamycin and Ceftriaxone. However, none of the isolates was sensitive to the Ampicillin, Cefotaxime, Erythromycin and Vancomycin. This is shown in Figure 1.

\section{Discussion}

The prevalence of GBS among pregnant women attending antenatal care clinic in Usmanu Danfodiyo University Teaching Hospital, Sokoto of $3.8 \%$ is close to the $2.5 \%$ that was reported from Lome, Togo [13]. This may be due to the similarity in geographical settings, study design and culture technique. The prevalence is however, lower than what was reported by other cross-sectional studies done in Blantyre, Malawi (16.5\%) [3], Ethopia, [12] 19.5\% reported from Uganda. [11] and 28.4\% reported from Panara, Sao Paulo [18]. Though the studies from Ile-Ife and Enugu were also hospital based cross-sectional studies, however, in these studies the women were screened at 35 - 40 and 24 - 37 weeks of gestation respectively. This could have accounted for the higher prevalence in their studies than in the current study. The prevalence in this study is also much lower than the $20 \%$ - 30\% prevalence in a multi-centered and multi-ethnic study reported by the Centre for Disease Control (CDC). Probably, this was due to a larger sample size of 1702 and repeat sample collection at delivery. Our prevalence is also lower than $25.3 \%$ and $30 \%$ colonization rate reported by Sabayek et al. [19] and Salat et al. [20] though they used Islam culture media and PCR technic respectively. Although their technique has sensitivity similar to our method, but also in their study, the women were screened between 24 to 40 weeks of gestation which could have accounted for the higher prevalence.

\section{Sensitivity pattern}

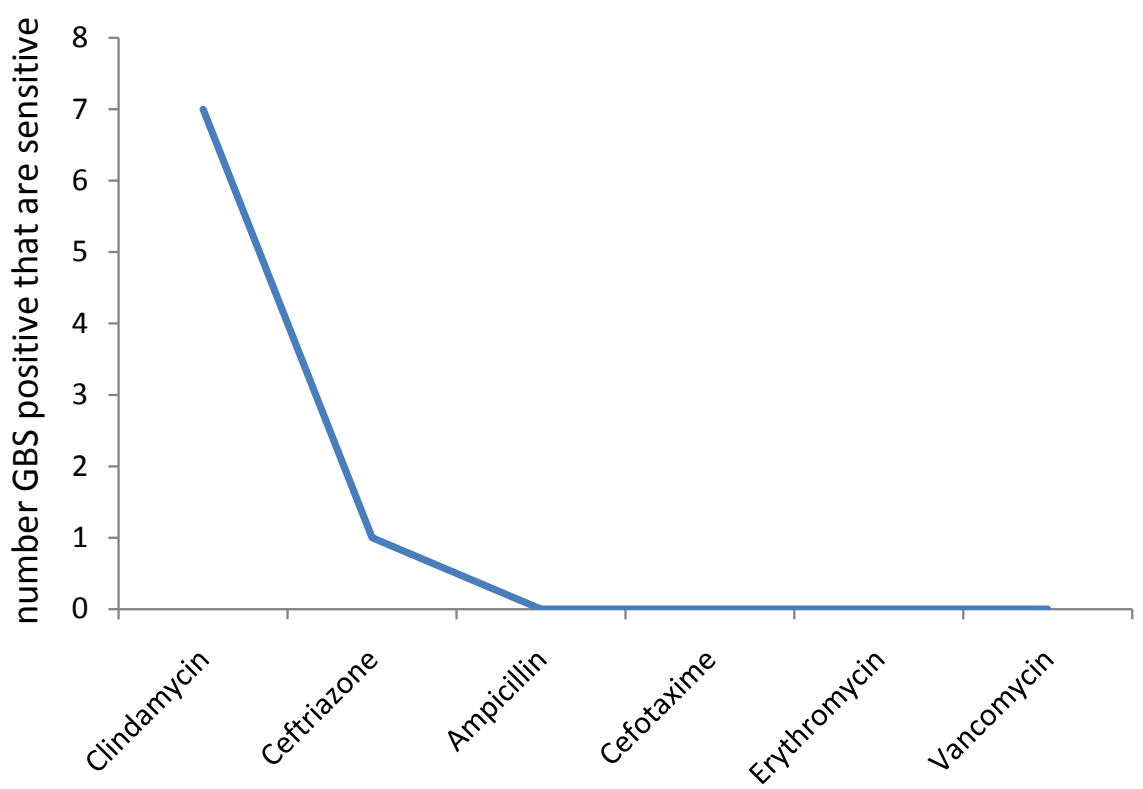

Figure 1. Drug sensitivity pattern of the GBS isolates. 
The level of GBS colonization was also found to be higher among women of low socio-economic class. This association could probably be due to the low level of personal hygiene in this group of women. This finding is consistent with multi-centre, multi-ethnic study from Netherland [10] as well as the study from Kenyatta National Hospital in 2008 by Salat et al. [20] which demonstrated relationship between GBS colonization and socio-economic background.

In relation to the parity and rate of GBS colonization among the study participants, the rate of colonization has been found to be higher in multi-parous women. However, the difference was not statistically significant. These findings are similar to what was found in a cross-sectional study done by Sabayak et al. [19] and Salat et al. [20] who found no association between GBS colonization and younger maternal age ( $>20$ years) and primigravidity. In their studies it was also found that GBS colonization is commoner among women of $\geq 30$ years and those with parity of $\geq 3$.

All the GBS isolates (100\%) in this study were sensitive to clindamycin and one is sensitive to both clindamycin and ceftriaxone. However, they were all (100\%) resistant to penicillin. This finding is similar to what was reported in a similar study done in Ile-Ife [14] which also showed that, all (17) their GBS isolates were $100 \%$ resistance to penicillin [14]. However, this is contrary to what was reported from Ethiopian [12] and Lomé, Togo [13] which revealed that all (100\%) of the GBS isolates were susceptible to penicillin G, vancomycin, ampicillin, erythromycin and gentamicin. Similarly, a study done among pregnant women in Nsukka, Enugu, Nigeria [13] and Mekkah, [21] demonstrated that all $(100.0 \%)$ of the isolates were sensitive to penicillin. However, in their study the sensitivity profile against Clindamycin was not tested. This finding of resistance to penicillin could as well be due to the frequent use of penicillin as over the counter (OTC) drug.

\section{Conclusions and Recommendations}

From this study, the GBS colonization rate among pregnant women attending antenatal care clinic at Usmanu Danfodiyo University Teaching Hospital, Sokoto was $3.8 \%$. There was an association between GBS colonization and low socio-economic status of the study participants. However, there was no association between GBS colonization and maternal age and low parity. It has also been found that previous bad pregnancy outcomes such as pre-mature rupture of membrane, pre-term delivery and stillbirth are not risks for GBS colonization among these studied participants. Clindamycin is an effective drug of choice in preventing maternal complication due to GBS colonization as well as preventing neonatal infection among babies GBS positive mothers in this environment.

A comparative study between urban and rural dwellers to compare rates of colonization between the two groups would give more information. Also a case control study among GBS negative women to compare clindamycin with other drugs to further substantiate its effectiveness as the drug of choice in preventing 
maternal complications among GBS positive women as well as preventing neonatal infection with GBS among the babies of GBS positive mothers.

\section{Conflicts of Interest}

The authors declare no conflicts of interest regarding the publication of this paper.

\section{References}

[1] Monica, C. (2006) Streptococcus agalactiae. In: Monica, C., Ed., District Laboratory Practice in the Tropics, 2nd Edition, Cambridge University Press, Cambridge, 162163.

[2] Musa, M., Daniel, A. and Yimtubezinash, W. (2012) Prevalence of Group B Streptococcus Colonization among Pregnant Women Attending Antenatal Clinic of Hawassa Health Centre. The Ethiopian Journal of Health Development, 26, 36-42.

[3] Dzowela, T., Komolafe, O.O. and Igbigbi, A. (2005) Prevalence of Group B Streptococcus Colonization in Ante Natal Women at the Queen Elizabeth Central Hospital, Blantyre-A Preliminary Study. Malawi Medical Journal, 17, 97-99.

https://doi.org/10.4314/mmj.v17i3.10888

[4] Patrik, D. (2001) Genital Tract Infection in Prregnancy. In: Frank, W.L. and Patrick, D., Eds., Obstetrics and Gynaecology; the Principles of Practice, McGrew Hill, New York, 103-104.

[5] Shobha, H.M. and Robert, J.S. (2013) Assessment of At-Risk Pregnancy. In: Alan, N.D., Lauren, N., Neri, L. and Ashley, S.R., Eds., Current Diagnosis and Treatment in Obstetrics and Gynecology, 11th Edition, McGrew Hill, New York, 228.

[6] Konar, H. (2010) Medical and Surgical Illness Complicating Pregnancy. In: Hiralal, K., Ed., DC Dutta's Textbook of Obstetrics Including Perinatology and Contraception, 7th Edition, New Central Book Agency Ltd., London, 296.

[7] Rooyen, T.M., Jan, E.A. and Berit, S. (2010) Group B Streptococcus Colonization during Pregnancy and Maternal-Fetal Transmission in Zimbabwe. Acta Obstetricia et Gynecologica Scandinavica, 89, 250-255.

https://doi.org/10.3109/00016340903398029

[8] Arijaan, W., Valkenburg, B., Arwen, J.S. and Paul, M.O. (2006) Prevalence of Colonization with Group B Streptococci in Pregnant Women of a Multi-Ethnic Population in the Netherlands. European Journal of Obstetrics \& Gynecology and Reproductive Biology, 124, 178-183. https://doi.org/10.1016/j.ejogrb.2005.06.007

[9] Melissa, K.V.D. (2009) Evaluation of Universal Antenatal Screening for Group B Streptococcus. The New England Journal of Medicine, 360, 2626-2636.

https://doi.org/10.1056/NEJMoa0806820

[10] Najmi, N., Sikandar, R., Zuberi, N.F. and Jehan, I. (2013) Maternal Genital Tract Colonization by Group B Stretococcus: A Hospital Based Study. Journal of Pakistan Medical Association, 63, 1103-1107.

[11] Clouse, K., Shehabi, A., Suleimat, A.M., Faouri, S., Khuri-Bulos, N., Al Jammal, A., Chappell, J., et al. (2019) High Prevalence of Group B Streptococcus Colonization among Pregnant Women in Amman, Jordan. BMC Pregnancy and Children, 177, 19. https://doi.org/10.1186/s12884-019-2317-4

[12] Alemseged, G., Selam, N., Haftamu, H., Mehamud, A., Muthupandian, S. and Tsehaye, A. (2015) Isolation and Anti-Microbial Susceptibility Pattern of Group B Streptococcus among Pregnant Women Attending Antenatal Clinic in Ayder Referral 
Hospital and Makelle Health Centre, Makelle, Nothern Ethopia. BMC Research Notes, 8, 518. https://doi.org/10.1186/s13104-015-1475-3

[13] Ezeonu, I.M. and Agbo, M.C. (2014) Incidence and Anti-Microbial Resistance Profile of Group B Streptococcus (GBS) Infection in Nsukka, Enugu, Nigeria. African Journal of Microbiology Research, 8, 91-95. https://doi.org/10.5897/AJMR12.2307

[14] Onipede, A., Adefusi, O., Adeyemi, A., Adejuyigbe, E., Oyelese, A. and Ogunniyi, T. (2012) Group B Streptococcus Carriage during Late Pregnancy in Ile-Ife, Nigeria. African Journal of Clinical and Experimental Microbiology, 13, 135-143. https://doi.org/10.4314/ajcem.v13i3.2

[15] Nwachukwu, N., Utsalo, S., Kanu, I. and Anyanwu, E. (2006) Genital Colonization of Group B Streptococcus at Term Pregnancy in Calabar, Nigeria. International Journal of Pediatrics and Neonatology, 7, 2. https://doi.org/10.5580/264c

[16] Daniel, W.W. (1999) Biostatistics: A Foundation for Analysis in the Health Sciences. 7th Edition, John Wiley and Sons, New York.

[17] Katz, V. and Bowes, W.A. (1988) Perinatal Group B Streptococcal Infections across Intact Amniotic Membranes. The Journal of Reproductive Medicine, 33, 445-449.

[18] de Melo, S.C.C.S., Costa, A.B., da Silva, F.T.R., Silva, N.M.M.G., Tashima, C.M., Cardoso, R.F., de Pádua, R.A.F., et al. (2018) Prevalence of Streptococcus agalactiae Colonization in Pregnant Women from the 18th Health Region of Paraná State. The Revista do Instituto de Medicina Tropical de São Paulo, 60, 212-218.

[19] Shabayek, S.A., Abdalla, S.M. and Abouzeid, A.M. (2009) Vaginal Carriage and Antibiotic Susceptibility Profile of Group B Streptococcus during Late Pregnancy in Ismailia, Egypt. Journal of Infection and Public Health, 2, 86-90. https://doi.org/10.1016/j.jiph.2009.03.004

[20] Gerald, K. (2007) Epidemiology of Early-Onset Neonatal Group B Streptococcal Infection; Implication for Screening. Canadian Family Physician, 53, 1054.

[21] Khan, M.A., Faiz, A. and Ashshi, A.M. (2015) Maternal Colonization of Group B Streptococcus: Prevalence, Associated Factors and Antimicrobial Resistance. Annals of Saudi Medicine, 35, 423-427. https://doi.org/10.5144/0256-4947.2015.423 


\section{Appendix 1}

QUESTIONAIRE

RISKS FOR GROUP B STREPTOCOCCAL COLONIZATION, PREVALENCE, RISK FACTORS AND DRUGS SENSITIVITY AMONG PREGNANT WOMEN IN USMANU DANFODIYO UNIVERSITY TEACHING HOSPITAL SOKOTO

\section{There respondents}

This questionnaire on the above subject is purely for academic purpose. All information obtained will be kept confidential.
A. SOCIO-DEMOGRAPHIC DATA
1) $S / N o$.
2) Phone No.
3) Hosp No
4) Patient phone no
5) Age....
6) Address.
7) Occupation:
a) Unemployed
b) Civil servant
c) Trader
d) Business woman

8) Tribe:
a) Hausa/Fulani
b) Yoruba
c) Igbo
d) Others (please specify).

9) Religion:
a) Islam
b) Christianity
c) Others (please specify)........

10) Level of Educational
a) No formal education
b) primary
c) Secondary
d) Tertiary

11) Marital status:
a) Single
b) Married
c) Divorced

12) Family setting:
a) Monogamous setting
b) Polygamous setting

13) Husband's Educ. Status: 

a) No formal education
b) Primary
c) Secondary
d) Tertiary

14) Husband's Occupation:
a) Civil servant
b) Trader
c) Driver
d) Businessman
e) Self employed

B. OBSTETRIC HISTORY

15) Parity;
a) primigravidae
b) multigravidae
c) grand-multipara

16) LMP EDD. EGA

17) History of PROM
a) Yes
b) No

18) History of Pre-term birth
a) Yes
b) No

19) History of sill birth
a) Yes
b) No

C. MICROBIOLOGICAL ANALYSIS

20) HVS
a) Microscopy.
b) Culture
c) Sensitivity (drug(s) 